\title{
Flexible, dynamic piezoresistive sensor matrix based on carbon nanotube polymer composite for pressure distribution measurement
}

\author{
Carina Gerlach, Abdulkadir Sanli, Rajarajan Ramalingame, Olfa Kanoun \\ Technische Universität Chemnitz, Reichenhainer Straße 70, 09126 Chemnitz, \\ carina.gerlach@etit.tu-chemnitz.de
}

\begin{abstract}
:
In this contribution, a large area piezoresistive sensor matrix based on a carbon nanotube polymer composite is shown for pressure distribution measurement applications. With an overall height of less than $200 \mu \mathrm{m}$ those sensitive films can measure pressure directly and therefore opens new fields of applications of pressure sensors were less available space is crucial. Besides the measurement of pressure, different shapes of loads can be detected as well. The method of a compact and noncomplex data acquisition system using a combination of LabVIEW and Arduino enables fast reading, which allows dynamic pressure measurement, e.g. during walking for an insole application.
\end{abstract}

Key words: piezoresistive sensor, mechanical sensor, sensor matrix, pressure distribution, carbon nanotube polymer composite.

\section{Motivation}

There is a need for thin and flexible sensor matrices for the measurement of pressure distribution, e.g. in plantar pressure distribution measurement to monitor critical pressure values as insole in every-day use [1] or as tactile sensitive skin in robotics [2], to name but a few. Moreover, the detection of different shapes is important in different fields of applications, e.g. the detection of a fallen and procumbent person [3] with a sensor matrix on the floor with differentiation of other objects at the same time [4] for ambient assisted living systems.

\section{Method}

In those applications, conventional silicon based pressure sensors get to their limit due to less available space. To overcome this issue polymer based films containing conducting filler particles are a promising material to realize piezoresistive sensors with less height, which are printable and therewith low-cost in fabrication as well as scalable in different dimensions ranging from single sensor to largearea sensor matrix. In comparison with capacitive sensors, piezoresistive sensors are more sensitive to mechanical stress [5], require a less complex data acquisition system and are less sensitive to electro-magnetic noise [6].

\section{Pressure distribution measurement}

In [7] a piezoresistive sensor matrix design based on a screen printed carbon nanotube polymer based film is proposed and was realized in laboratory scale. Furthermore, the sensor fabrication was explained.

In this paper, we present an enhanced sensor design, whereat the electrode matrix is applied on a flexible and temperature stable polyimide foil, seen in Fig. 1, on which the sensor material is placed.

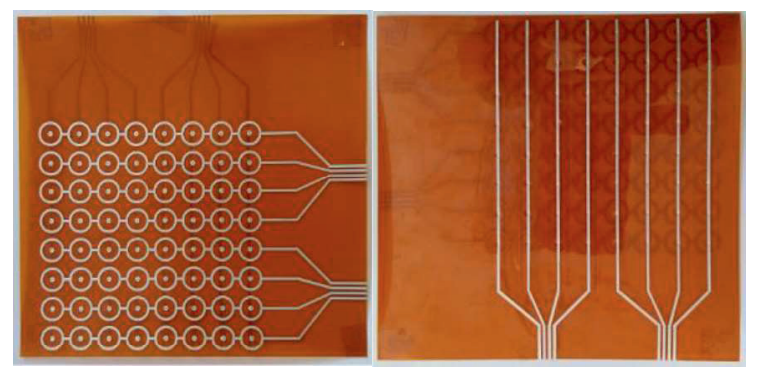

Fig. 1. Electrode matrix design: silver electrodes on polyimide foil (top and bottom view)

Moreover, the manual addressing is replaced by an automatic addressing and reading data acquisition system based on a combination of LabVIEW and Arduino, shown schematically in Fig. 2.

Thereby, a faster signal processing is achieved, which enables dynamic measurements, like the detection of pressure distribution during 
walking. Furthermore, different sizes and positions of circular shaped samples can be detected, seen as conductivity matrix in Fig. 3.

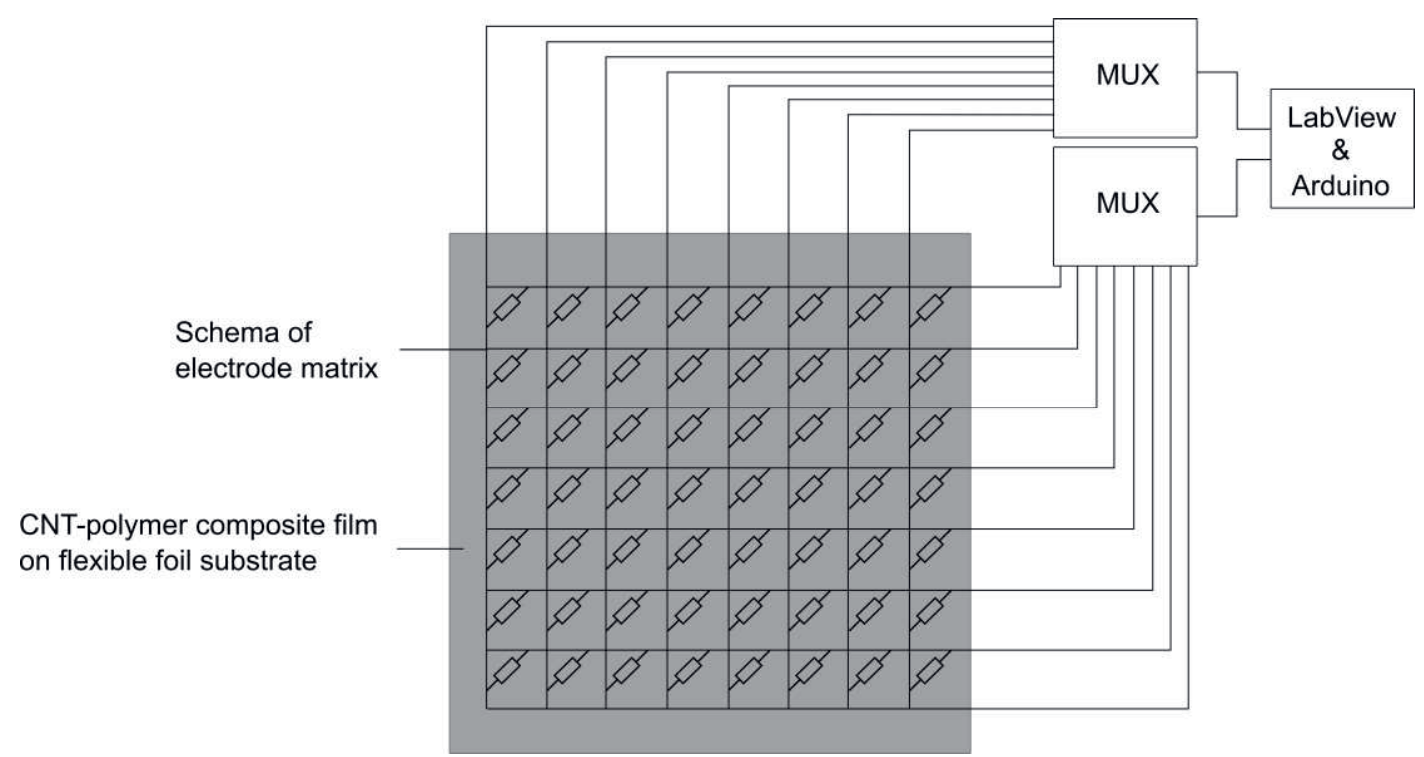

Fig.2. Schematic representation of the data acquisition system of localized pressure and pressure distribution measurement.
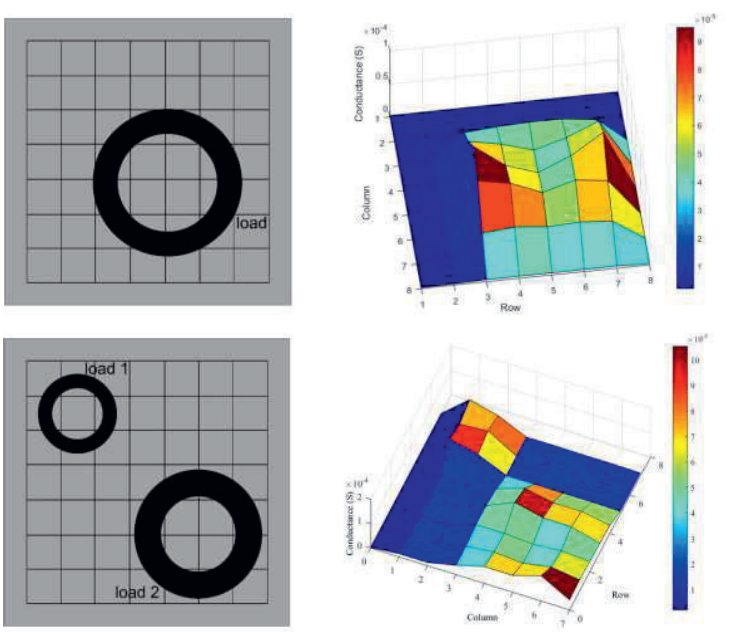

Fig.3. Exemplary pressure distribution measurements of different sizes and positions of circular rings measured with an $8 \times 8$ sensor matrix $(10 \times 10 \mathrm{~cm})$ showed as conductance matrix.

\section{Conclusion}

A thin piezoresistive sensor matrix based on carbon nanotube polymer composite as pressure sensor on flexible substrate was realized. Thereby pressure distribution measurements and load detection of different shapes are possible. With a data acquisition system based on LabVIEW and Arduino a dynamically measurement is achieved.

\section{References}

[1] J. Gurney, U. G. Kersting, D. Rosenbaum. Gait \& posture, 27.4 (2008): 706-709; doi: 10.1016/j.gaitpost.2007.07.002

[2] T. Someya, Y. Kato, T. Sekitani, S. Iba, Y. Noguchi, Y. Murase, H. Kawaguchi, T. Sakurai. Proceedings of the National Academy of Sciences of the United States of America 102.35 (2005): 12321-12325; doi: 10.1073/pnas.0502392102

[3] C. Lauterbach, A. Steinhage, A. Techmer. $9^{\text {th }}$ International Multi-Conference on Systems, Signals and Devices (2012), Chemnitz; doi: 10.1109/SSD.2012.6198101

[4] A. Mihai, O. Simonin F. Charpillet. IEEE Sensors Journal, 16.4 (2016): 1026-1037; doi: 10.1109/JSEN.2015.2493122

[5] M. Amjadi, K. Kyung, I. Park, M. Sitti. Advanced Functional Materials, 26 (2016): 1678-1698; doi: 10.1002/adfm.201504755

[6] Z. Kappassov, J.-A. Corrales, V. Perdereau. Robotics and Autonomous Systems 74 (2015): 195-220; doi: 10.1016/j.robot.2015.07.015

[7] C. Gerlach, D. Krumm, M. Illing, J. Lange, O. Kanoun, S. Odenwald, A. Hübler. IEEE Sensors Journal 15.7 (2015): 3647-3656; doi: 10.1109/JSEN.2015.2392084 